\title{
Release of small hydrophilic molecules from polyelectrolyte capsules: Effect of the wall thickness
}

\author{
Francesca Cuomo $^{\mathrm{a}, *}$, Francesco Lopez ${ }^{\mathrm{a}}$, Marco Piludu ${ }^{\mathrm{b}}$, Maria G. Miguel ${ }^{\mathrm{c}, \mathrm{e}}$, Bjorn Lindman ${ }^{\mathrm{d}, \mathrm{e}, \mathrm{f}}$, \\ Andrea Ceglie ${ }^{\mathrm{a}}$ \\ a Dipartimento di Agricoltura, Ambiente Alimenti (DIAAA) and CSGI, Università degli Studi del Molise, Via De Sanctis, I-86100 Campobasso, Italy \\ ${ }^{\mathrm{b}}$ Dipartimento di Scienze Biomediche, Università di Cagliari, Cittadella Universitaria, S.S. 554 bivio Sestu, 09042 Monserrato, CA, Italy \\ ${ }^{\mathrm{C}}$ Chemistry Department, Coimbra University, 3004-535 Coimbra, Portugal \\ d Physical Chemistry, Lund University, P.O. Box 124, 22100 Lund, Sweden \\ ${ }^{\text {e }}$ School of Materials Science \& Engineering, Nanyang Technological University, Singapore \\ ${ }^{\mathrm{f}}$ FSCN, Fibre Science and Communication Network, MidSweden University, Sundsvall, Sweden
}

\section{A R T I C L E I N F O}

\section{Article history:}

Received 25 September 2014

Accepted 24 October 2014

Available online 4 November 2014

\section{Keywords:}

Layer by layer

Polyelectrolyte nanocapsules

Multishell thickness

Molecule release

\begin{abstract}
A B S T R A C T
Polymer nanocapsules assembled on cationic liposomes have been built through the layer-by-layer (LbL) technique. Chitosan and alginate, two biocompatible polyelectrolytes, were used to cover the template, where the Rhodamine B was previously loaded. The multishell formed with the alternate deposition of the polyelectrolytes, according to the principles of the LbL assembly, was supposed to change the permeability of the capsule wall. The thickness of the multishell was seen increasing with the number of layers deposited through the observations with the Transmission Electron Microscope. The permeability of the capsules was studied through Rhodamine B release assays. Nanocapsules with seven layers of polyelectrolytes released the dye slowly compared to the capsules with three or five layers. The Ritger-Peppas model was applied to investigate the release mechanisms and a non-Fickian transport behavior was detected regardless of the number of layers. Values of diffusion coefficients of Rhodamine B through the capsule wall were also calculated.
\end{abstract}

(c) 2014 Elsevier Inc. All rights reserved.

\section{Introduction}

In the last few years, the development of multi-compartmental tools is providing delivery devices especially relevant in the fields of medicine and biology [1-7]. A great number of them is designed by taking advantage of the self-assembly properties of the constituents [8-14]. Among the emerging materials the polymer capsules are drawing great attention. They are considered as challenging devices because of their unique structural properties. Polymer capsules are structures having mainly spherical shape and are produced by coating templates made of different materials using different strategies [15-17]. One of the approaches generally recognized as effective in the assembly of polymer capsules is the layer-by-layer technique (LbL) $[18,19]$. This technique is based on the alternate deposition of oppositely charged polyelectrolytes onto templates of different size and composition [20-23]. The main driving force for LbL assembly is the electrostatic interaction among templates and polyelectrolytes. The electrostatic attraction,

\footnotetext{
* Corresponding author.

E-mail address: francesca.cuomo@unimol.it (F. Cuomo).
}

in fact, rules the polyelectrolyte association and, the repulsion between assemblies with the same surface charge is essential to the stability of the assembled micro- or nano-sized containers. The alternate deposition of polyelectrolytes generates a polymer wall surrounding the template, which can be removed thus leaving a spherical volume, generally filled with water, which remains separated from the external environment thanks to the presence of the polymer multishell. According to the type of polyelectrolytes assembled and to the assembly method, new materials are formed having different properties from those offered by the individual polymers. The matrix formed by the polymer assembly is a network that controls the permeability of the capsule wall. Permeability can be modified varying the number of assembled layers or the number of interactions between the polymers, for instance, by changing the $\mathrm{pH}$ or the ionic strength [24-28].

Kinetics studies on molecular release from these devices represent a relevant aspect to evaluate the utility of such systems as drug delivery devices in biological and biomedical applications. Drug encapsulation is, therefore, another key point. Polymeric capsules can be filled with drugs before the layer deposition or after the core removal. In some cases the LbL procedure is applied to micro- or nano-dispersions of drugs [24,29]. 
The interest in the use of compartmentalized systems as drug delivery devices is due to the opportunity they offer of enhancing the therapeutic efficacy and of reducing the dose of drug administered and, consequently, the side effects of a drug therapy [22,30-32].

The production of containers loaded with drugs is mainly directed towards the realization of drug delivery systems having dimensions in the nanometer range because nano-sized systems should remain in the circulation for longer times compared to the micro-sized particles, which are subject to clearance via the lymphatic system [33]. Therefore, the biological activity of nano-systems would be of a higher extent [34].

Very recently, Łukasiewicz and Szczepanowicz [35] demonstrated that nanocapsules assembled on a liquid oil core stabilized by AOT (dioctyl sodium sulfosuccinate) and covered with polyelectrolyte shells of PLL (poly-L-lysine) and PGA (polyL-glutamate) are quite safe for use as delivery vehicles. Parekh et al. [36] produced nanocapsules made of heparin and a blockcopolymer of poly-l-lysine and polyethylene glycol, containing camptothecin in the core. In order to prevent the clearance of nanocapsules in the circulation, the outer surface of the nanocapsules was modified with polyethylene glycol of $5 \mathrm{kDa}$ or $20 \mathrm{kDa}$ molecular weight. These authors demonstrated that these LbL-coated capsules are effective in the preservation of the lactone form of camptothecin, which is the active form of the drug. Thomas et al. [37] investigated the release of doxorubicin encapsulated in hollow biocompatible nanocapsules made of chitosan/heparin assembled onto $\mathrm{SiO}_{2}$ nanoparticles. The doxorubicin encapsulated was successfully internalized in MCF-7 cell lines and in vivo experiments on BALB/c mice it was revealed that the drug circulation time was increased compared with free doxorubicin. Yan et al. [38] demonstrated the loading and sustained release of 5-fluorouracil, a hydrophobic molecule, in microcapsules made of poly (L-glutamate)/chitosan assembled onto melamine formaldehyde (MF) templates. The drug was encapsulated into the capsule core after template dissolution.

Among the most used models for quantitative studies of drug release profiles from particles there are the Higuchi model, the Ritger-Peppas model, the Weibull function, and the Hixson-Crowell model. All of them take into consideration the relation between the structure and the function of the device, the release environment, and the possible interactions between the wall of the device and the drug molecules $[39,40]$. These models describe the transport behavior as strictly related to the Fick's law of diffusion and suggest that the description of solute transport from polymeric matrices can be divided into two categories: Fickian and non-Fickian behavior.

Recently, we proposed a way for producing hollow nanocapsules by covering a liposome core with biocompatible polyelectrolytes, namely chitosan and alginate, via the LbL self-assembly method [25,26] and, moreover, we have demonstrated that these structures can be used as drug reservoirs of hydrophilic molecules with high molecular weight on the basis of release assays from liposome based polymer nanocapsules [41].

In the present study, the investigation is extended to the encapsulation and release from liposome based polymer nanocapsules of a small hydrophilic molecule as a function of the multishell thickness.

\section{Materials and methods}

\subsection{Materials}

L- $\alpha$-phosphatidylcholine (egg yolk lecithin) was purchased from Avanti Polar. Lipids, didodecyldimethylammonium bromide (DDAB), sodium chloride, low molecular weight sodium alginate, low molecular weight chitosan and Triton X-100, Rhodamine B and dialysis tubing cellulose membranes, with a molecular weight cut off at 3.5-5 kDa and at $100 \mathrm{kDa}$, were purchased from SigmaAldrich.

\subsection{Preparation of liposome-templated chitosan/alginate nanocapsules}

Unilamellar liposomes $(80 \mathrm{~nm})$ were prepared by reversed phase evaporation according to the method described by Szoka and Papahadjopoulos [42] with a 6.5:3.5 M mixture of phosphatidylcholine and DDAB. Lipid and surfactant were dispersed in $3 \mathrm{~mL}$ of diethyl ether and to this $1 \mathrm{~mL}$ of PBS buffer ( $\mathrm{pH} \mathrm{7.2)} \mathrm{con-}$ taining $0.5 \mathrm{mg} / \mathrm{mL}$ of Rhodamine $\mathrm{B}$ was added, forming a twophase system, which was mixed by means of a sonicator tip to form a dispersion of inverted micelles. A rotary evaporator removed the organic solvent, thus, the inverted micelles became an aqueous suspension of liposomes. Finally, additional two milliliters of buffer were added and the suspension was left for further $45 \mathrm{~min}$ at the rotary evaporator to remove any trace of solvent. The final lipid concentration was $16 \mathrm{mg} \mathrm{mL}^{-1}$ (i.e., $20 \mathrm{mM}$ ). The liposomes were then sequentially extruded through 0.1 and $0.05 \mu \mathrm{m}$ polycarbonate membranes before use. The excess of fluorescent dye was removed through a dialysis membrane ( $12 \mathrm{~h}$ ) with a cut-off of $100 \mathrm{kDa}$ against $500 \mathrm{~mL}$ of buffer solution (PBS pH 7.2). The procedures of chitosan/alginate deposition onto the surfaces of colloidal particles, as well as lipid core removal, were reported elsewhere [26].

\subsection{Transmission Electron Microscopy}

TEM high resolution characterization of the samples was carried out placing appropriately diluted drops of each suspension on formvar coated 100 mesh size grids for 5 min. Excess of suspension was adsorbed touching the edge of the grids with filter paper. Finally, the grids were air dried, observed and photographed with a JEOL 100S Transmission Electron Microscope.

\subsection{Rhodamine B encapsulation efficiency}

The encapsulation efficiency (EE\%) of Rhodamine B into liposomes and into core-shell capsules was calculated as follows:

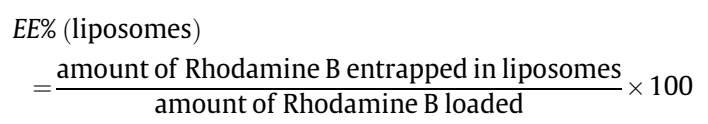

EE\% (core-shell)

actual amount of Rhodamine B entrapped in core-shell capsules theoretical amount of Rhodamine B in core-shell capsules $\times 100$

The theoretical concentration of Rhodamine $\mathrm{B}$ in core-shell capsules was calculated as the expected concentration of the dye after the dilution of dye entrapped into liposomes after the polyelectrolyte deposition.

\subsection{Rhodamine B release}

The samples were dialyzed through a membrane with a cut off of $100 \mathrm{kDa}$ to follow the release of Rhodamine B. The bulk dialysis was prepared according to the ionic strength of the sample and with PBS $\mathrm{mM}$ at $\mathrm{pH}$ 7.2. The samples were left in the bulk to equilibrate for half an hour and in this time window we did not observe any change in Rhodamine B concentration. Subsequently a volume of $100 \mu$ of $11 \mathrm{mM}$ Triton X-100 solution was added to the sample 
for liposome rupture and after 20 min the sample was placed again in the dialysis tube. The time of $20 \mathrm{~min}$ was long enough for Triton $\mathrm{X}-100$ to interact with liposome inducing vesicle-to-mixed micelles transition [26]. At specific intervals samples were withdrawn for spectrophotometric analysis, using a Cary 100 Bio UV/Vis spectrophotometer from Varian. Spectra were collected in the 800-200 nm range, at $25^{\circ} \mathrm{C}$, using quartz cells with a path length of $1 \mathrm{~cm}$. Absorption spectra were fitted, in the spectral region between 300 and $450 \mathrm{~nm}$, with a power law: $A=K_{0} \lambda^{-k}$, where $\lambda$ is the wavelength in the range defined above, whereas $k$ (for Rayleigh scatterers $k \approx 4$ ) and $K_{0}$ are adjustable parameters. Then, the calculated scattering curve has been extrapolated to $220 \mathrm{~nm}$ and finally subtracted from the measured absorption spectrum. The absorbance values collected were converted into concentration values according to the calibration curve previously established.

\section{Results and discussions}

Applying the LbL procedure, core-shell capsules were assembled. Cationic liposomes were covered with alginate and chitosan and the morphology of the capsules was observed by Transmission Electron Microscopy.

Photographs of core-shell capsules with three, five and seven layers are reported in Fig. 1. The mean dimension of the core-shell particles is $250 \mathrm{~nm}$; the particles are quite polydisperse as also shown in the figure. The capsules loose their spherical shape because they are dried before the TEM observation. At the same time, however, the particle shell has a constant thickness in all the structures visible in Fig. 1A where the particles have three layers of polyelectrolytes. The values of multishell thickness were calculated by measuring the bar-dimension length of the TEM photograph and comparing its length to the length of the capsule wall. The mean values are calculated from observations on ten capsules from three different batches.

A constant and thicker profile can be seen for the core-shell structures with five layers, in Fig. 1B and, finally, a further increase of the shell thickness is recognizable around the structures with seven polyelectrolyte layers reported in Fig. 1C.

The mean values of the multishell thickness are indicated in Fig. 2, where it seems that a linear increase of thickness is given by the addition of layers. As shown in the graph, the shell thickness is $15 \mathrm{~nm}$ with three layers, $20 \mathrm{~nm}$ with five layers and $25 \mathrm{~nm}$ with seven layers. This parameter is of fundamental importance for studies of molecular release from the inner core of the capsules.

In order to study the release of small hydrophilic molecules from the assembled nanocapsules, Rhodamine B was pre-encapsulated into liposomes that were successively covered with polyelectrolyte layers and, the release of the dye was analyzed from samples with three, five and seven layers as well as from the bare liposomes.

The retention of Rhodamine B in the liposome template was about $32.5 \%$. After the layer deposition, the amounts of Rhodamine encapsulated into the core-shell structures with three, five and seven layers were about $37.5 \%(1.11 \mu \mathrm{M}), 41 \%(1.04 \mu \mathrm{M})$ and $43 \%$ $(0.95 \mu \mathrm{M})$ of the expected concentrations respectively. The expected concentration of dye was the theoretical concentration calculated from the dilution that the dye entrapped into liposomes required with the polyelectrolyte deposition. This decrease can be ascribed to the procedure of preparation of core-shell particles. The samples are indeed centrifuged after the deposition of each layer to eliminate the macroaggregates eventually formed after the polyelectrolyte addition.

The event that triggered the release of Rhodamine $B$ was the addition of the nonionic surfactant Triton X-100, which, interacting with liposomes, caused their dissolution and made the loaded dye,
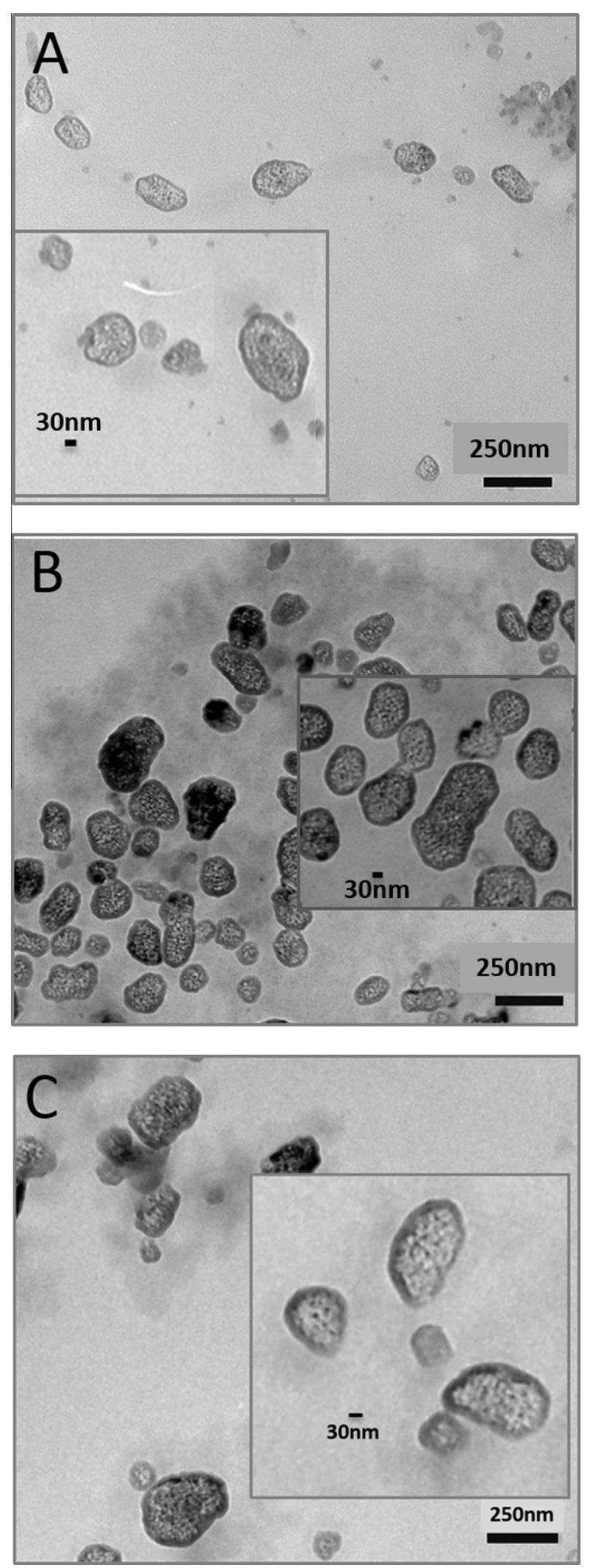

Fig. 1. TEM photographs of core-shell capsules covered with three (A), five (B) and seven (C) layers of polyelectrolytes. Insets in panels $A-C$ are magnifications of the respective photographs.

Rhodamine B, free to diffuse from the aqueous core of the nanocapsules through the polyelectrolyte multishell. After the liposome removal, the structure of the capsules remains unchanged as demonstrated earlier $[25,26,41]$. The release proofs were realized by dialysis, where $1 \mathrm{~mL}$ of nanocapsules suspension enclosed in a dialysis membrane (cutoff of $100 \mathrm{kDa}$ ) was placed into bulk made of a 


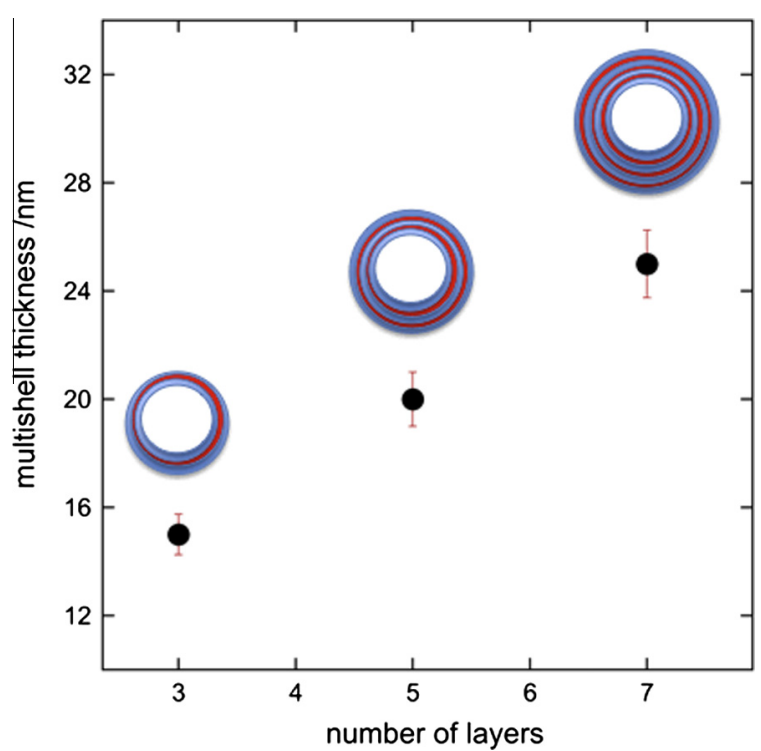

Fig. 2. Variation of the multishell thickness as a function of the number of polyelectrolyte layers. The values of thickness are obtained from the TEM images.

buffer solution at $\mathrm{pH} 7.2$ having the same ionic strength as the sample. In Fig. 3 the time-dependent release profiles of Rhodamine $\mathrm{B}$ from bare liposomes and from core-shell nanocapsules with three layers are reported as a function of time.

In both the samples the release process started after the addition of Triton X-100. In a comparison of the release profiles it is evident that the presence of polyelectrolyte layers offers a barrier to the escape of the loaded dye, in fact, after the first 30 min the amount of Rhodamine B released from bare liposomes is about $27 \%$ and the same amount is released from core-shell nanocapsules in $1 \mathrm{~h}$. Additionally, after two hours $74 \%$ of the loaded molecules is released from liposomes while $48 \%$ is liberated from core-shell nanocapsules. In Fig. 4, the time dependent release profiles are shown from nanocapsules having three, five and seven layers. From this chart, at first glance, it is evident that the release profiles for structures with three and five layers are quite similar, while a slower release is

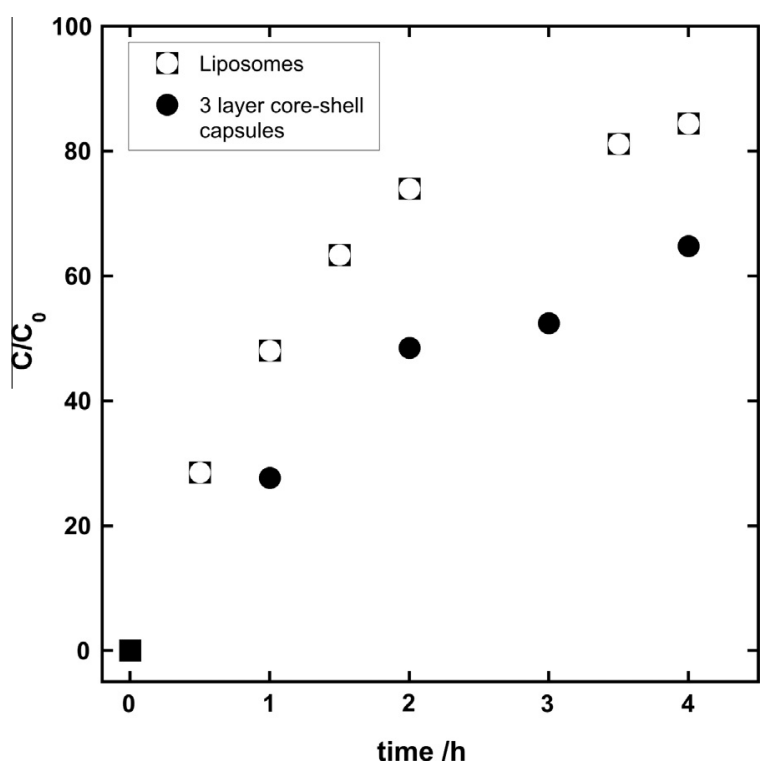

Fig. 3. Percentage release of Rhodamine B from liposomes (empty circles) and from core-shell capsules with three layers (black circles) after the addition of Triton X100. detected for nanocapsules with seven layers. As seen from the release profiles, it is noticeable that after $1 \mathrm{~h}$ the capsules with three or five layers can leave $27 \%$ of their content whereas capsules with seven layers need about $4 \mathrm{~h}$ to release the same amount of dye.

To investigate more precisely the mechanism of Rhodamine $B$ release from nanocapsules, the experimental data were analyzed according to the Ritger-Peppas equation, a power law used to describe the general solute release behavior from different polymeric devices [43-45]:

$\frac{C}{C_{0}}=k t^{n}$

In this relation, $C / C_{0}$ is the fractional amount of the drug released at time $t, n$ is a diffusion exponent indicating the release mechanism, and $k$ is a characteristic constant of the system. In the case of a sphere, values of $n$ lower than 0.43 are found for a Fickian diffusion release mechanism; values of $n$ in a range between 0.43 and 1 indicate anomalous non-Fickian transport. The experimental release profiles have been fitted to Eq. (1) and the results are reported in Fig. 5A. The values of $n$ deriving from fittings are represented in Fig. 5B where the line boundary in the figure is placed at the $n$ value of 0.43 to underline the limit between Fickian and non-Fickian behavior.

The calculated $n$ values indicate that only bare liposomes release Rhodamine B with a Fickian transport mechanism. On the other side, values of $n$ for nanocapsules with three or five layers are very close to one another and slightly higher than the threshold value; the $n$ value for seven layer nanocapsules is definitely higher. It is clear that, for the nanocapsules, a non-Fickian behavior rules the release mechanism, but this is more pronounced for a higher value of the number of polymer layers.

Comparing our results with other release studies from the literature, it seems that opposing behaviors are found from the release mechanism analysis. In fact, the $n$ values given by Yan et al. [38] suggested a Fickian type of transport for 5-fluorouracil released from poly (L-glutamic acid)/chitosan microcapsules. Nevertheless, 5-fluorouracil is a small molecule and its molecular weight is comparable with the one of Rhodamine B. Moreover, Shu et al. [44] observed a Fickian mechanism of transport for bovine serum albumin (BSA) from capsules of water-soluble

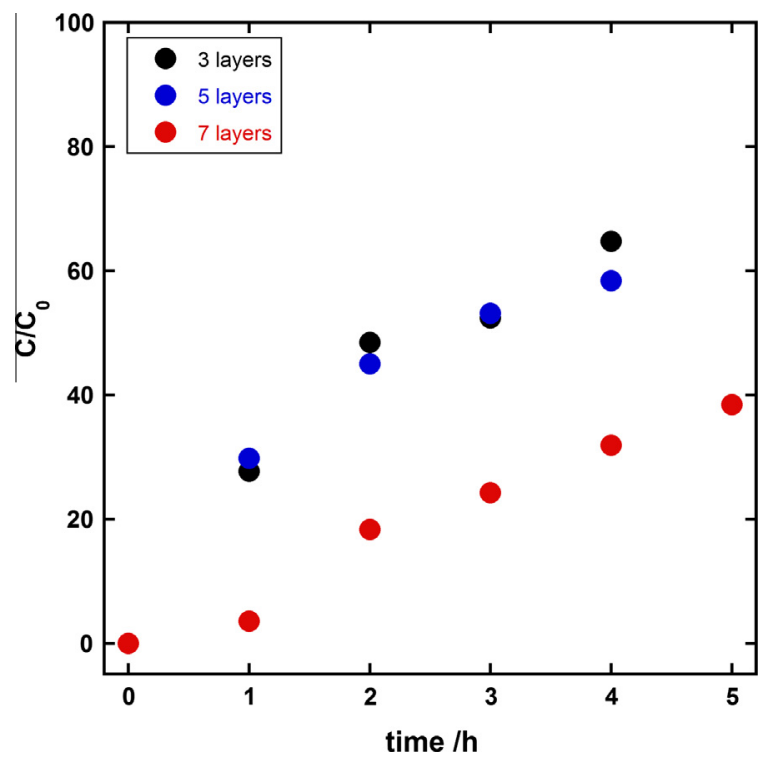

Fig. 4. Percentage release of Rhodamine B from core-shell capsules with three layers (black circles), five layers (blue circles) and seven layers (red circles) after the addition of Triton X-100. (For interpretation of the references to color in this figure legend, the reader is referred to the web version of this article.) 

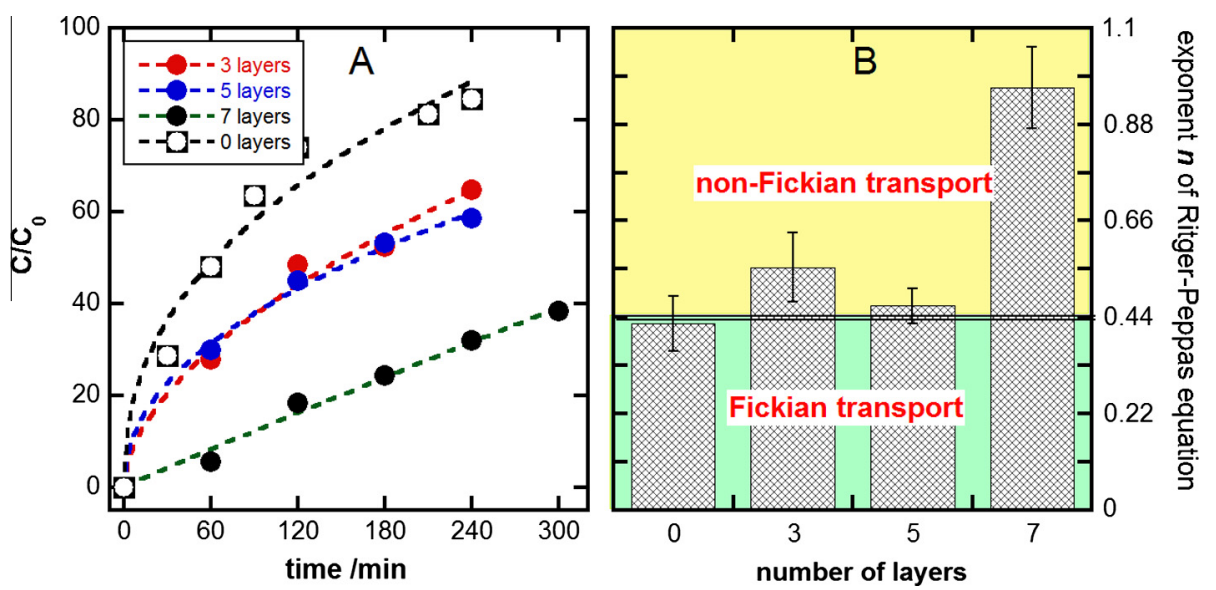

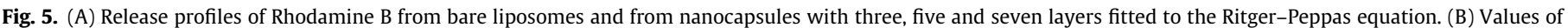

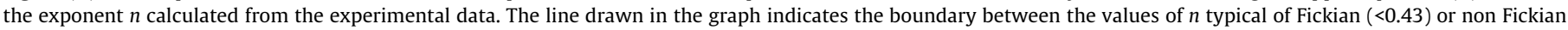
diffusion $(>0.43)$.

chitosan and dextran sulfate encapsulating the protein, despite the high molecular weight of the BSA. In a previous investigation, we explored the release of FITC-dextran molecules with different molecular weight from nanocapsules with five alternating layers of alginate and chitosan. The release mechanisms determined were a non-Fickian transport type for all the tested molecules [41]. All this considered, it could be deduced that every single system, loaded with different molecules, has its own peculiar release behavior depending on the assembly conditions and on the multishell composition.

Apparent diffusion coefficients of Rhodamine B, through the polyelectrolyte multishell, were calculated with the following equation, according to the model used for other similar systems $[46,47]$ :

$\frac{C}{C_{0}}=4\left(\frac{D_{a p p} t}{\pi h^{2}}\right)^{0.5}$

where $D_{a p p}$ is the apparent diffusion coefficient, $h$ is the multishell thickness, with values of about 15, 20 and $25 \mathrm{~nm}$ obtained from the TEM images for core-shell structures with three, five and seven

Table 1

Best-fit values calculated from fitting of the data to Eq. (2)

\begin{tabular}{lll}
\hline & $D_{a p p}\left(\times 10^{-15}\right)\left(\mathrm{cm}^{2} / \mathrm{s}\right)$ & $R^{2}$ \\
\hline 3 Layers & $6.7( \pm 0.4)$ & 0.9855 \\
5 Layers & $9.9( \pm 0.3)$ & 0.9955 \\
7 Layers & $0.66( \pm 0.14)$ & 0.8978 \\
\hline
\end{tabular}

layers respectively. Values of the apparent diffusion coefficient calculated from Eq. (2) are reported in Table 1.

The $D_{a p p}$ values shown in the table highlight the different diffusion coefficients of Rhodamine B from structures with seven layers compared to structures with five and three layers. According to the difference noticed in the release profiles there is a difference of one order of magnitude between the apparent diffusion coefficients of Rhodamine B from capsules with seven layers compared to the ones with three and five layers. It seems that the presence of a thicker multishell provides a barrier that slows down the molecule diffusion as represented in the sketch of Fig. 6. Moreover, these values are in agreement with the information collected earlier concerning the diffusion coefficients for the passage of dextran of FITC-dextran through five layer nanocapsules. In that case, it was observed that the diffusion coefficients were all much lower compared to those of Rhodamine B, and that the multishell wall worked as a filter for molecules having molecular weight of $40 \mathrm{kDa}$ or higher.

\section{Conclusion}

Liposome based polyelectrolyte nanocapsules have been assembled through the layer-by-layer (LbL) technique. Chitosan and alginate were used to cover the template. Transmission Electron Microscopy was used to investigate the variation of the thickness of the multishell with the number of layers deposited. The permeability of the capsules changes according to the wall thickness and the relation between these two parameters was studied through Rhodamine B release assays. Even capsules with only three layers

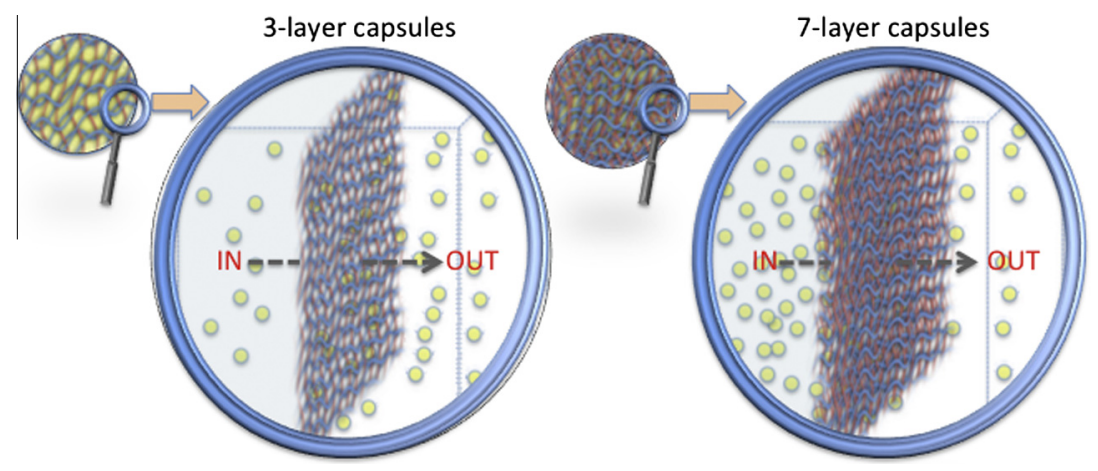

Fig. 6. Schematic representation of the effect of the multishell thickness on the molecule release. 
of polyelectrolytes are able to provide a barrier to the dye release, as seen from the comparison between the escape of Rhodamine B from bare liposomes and from three layer structures. Nanocapsules covered with seven layers of polyelectrolytes released the dye slowly compared to the capsules with three or five layers. From the data fitting to the Ritger-Peppas model the release transport was determined as non-Fickian type regardless of the number of layers. This finding seems to be in contrast with the results of other studies $[38,44]$, where either low or high molecular weight molecules were released with a Fickian type of transport. Nevertheless polymer capsules are so different to one another that every single system, loaded with different molecules, has its own peculiar release behavior depending on the dimension, on the assembly conditions and on the multishell composition. Values of diffusion coefficients of Rhodamine B through the capsule wall were also calculated, thus providing other additional information concerning the potential of these systems as drug delivery devices.

\section{Acknowledgments}

This work was financially supported by MIUR (PRIN 2010, 2010BJ23MN), CSGI and, Fondazione Banco di Sardegna.

\section{References}

[1] L.L. del Mercato, M.M. Ferraro, F. Baldassarre, S. Mancarella, V. Greco, R. Rinaldi, S. Leporatti, Adv. Colloid Interface Sci. 207 (2014) 139-154.

[2] G. Aguirre, J. Ramos, J.P.A. Heuts, J. Forcada, Polym. Chem. 5 (2014) 4569-4579.

[3] H. Wei, R.-X. Zhuo, X.-Z. Zhang, Prog. Polym. Sci. 38 (2013) 503-535.

[4] F. Cuomo, A. Ceglie, F. Lopez, J. Colloid Interface Sci. 365 (2012) 184-190.

[5] R. Angelico, A. Ceglie, P. Sacco, G. Colafemmina, M. Ripoli, A. Mangia, Int. J. Pharm. 471 (2014) 173-181.

[6] P. Rivera Gil, L.L. del Mercato, P. del-Pino, A. Munoz-Javier, W.J. Parak, Nano Today 3 (2008) 12-21.

[7] A. Cardone, F. Lopez, F. Affortunato, G. Busco, A.M. Hofer, R. Mallamaci, C. Martinelli, M. Colella, G.M. Farinola, Biochim. Biophys. Acta, Biomembr. 1818 (2012) 2808-2817.

[8] F. Cuomo, M. Mosca, S. Murgia, A. Ceglie, F. Lopez, Colloids and Surf., B 104 (2013) 239-244.

[9] F. Lopez, F. Cuomo, A. Ceglie, L. Ambrosone, G. Palazzo, J. Phys. Chem. B 112 (2008) 7338-7344.

[10] L. Piculell, B. Lindman, Adv. Colloid Interface Sci. 41 (1992) 149-178.

[11] J. Boekhoven, P. van Rijn, A.M. Brizard, M.C.A. Stuart, J.H. van Esch, Chem. Commun. 46 (2010) 3490-3492.

[12] O.A. Scherman, Nat. Chem. 1 (2009) 524-525.

[13] F. Cuomo, M. Mosca, S. Murgia, P. Avino, A. Ceglie, F. Lopez, J. Colloid Interface Sci. 410 (2013) 146-151.
[14] F. Cuomo, G. Palazzo, A. Ceglie, F. Lopez, J. Photochem. Photobiol. A 202 (2009) 21-27.

[15] F. Cuomo, F. Lopez, A. Ceglie, Adv. Colloid Interface Sci. 205 (2014) 124-133.

[16] J. Jose, M. Kamp, A. van Blaaderen, A. Imhof, Langmuir 30 (2014) 2385-2393.

[17] A.P. Esser-Kahn, S.A. Odom, N.R. Sottos, S.R. White, J.S. Moore, Macromolecules 44 (2011) 5539-5553.

[18] Z. Dai, A. Voigt, S. Leporatti, E. Donath, L. Dähne, H. Möhwald, Adv. Mater. 13 (2001) 1339-1342.

[19] G.B. Sukhorukov, E. Donath, H. Lichtenfeld, E. Knippel, M. Knippel, A. Budde, H. Möhwald, Colloids Surf., A 137 (1998) 253-266.

[20] B.V. Parakhonskiy, A.M. Yashchenok, M. Konrad, A.G. Skirtach, Adv. Colloid Interface Sci. 207 (2014) 253-264.

[21] Y. Tan, U.H. Yildiz, W. Wei, J.H. Waite, A. Miserez, Biomacromolecules (2013).

[22] K. Ariga, Y.M. Lvov, K. Kawakami, Q. Ji, J.P. Hill, Adv. Drug Delivery Rev. 63 (2011) 762-771.

[23] W.J. Tong, C.Y. Gao, Chem. J. Chin. U 29 (2008) 1285-1298.

[24] A. Agarwal, Y. Lvov, R. Sawant, V. Torchilin, J. Controlled Release 128 (2008) $255-260$.

[25] F. Cuomo, F. Lopez, A. Ceglie, L. Maiuro, M.G. Miguel, B. Lindman, Soft Matter 8 (2012) 4415-4420.

[26] F. Cuomo, F. Lopez, M.G. Miguel, B. Lindman, Langmuir 26 (2010) 1055510560.

[27] S. De Koker, L.J. De Cock, P. Rivera-Gil, W.J. Parak, R. Auzély Velty, C. Vervaet J.P. Remon, J. Grooten, B.G. De Geest, Adv. Drug Delivery Rev. 63 (2011) 748761.

[28] O.H. Zhao, B.Y. Li, Nanomed. Nanotechnol. 4 (2008) 302-310.

[29] S.J. Strydom, D.P. Otto, N. Stieger, M.E. Aucamp, W. Liebenberg, M.M. de Villiers, Powder Technol. 256 (2014) 470-476.

[30] H. Ai, Adv. Drug Delivery Rev. 63 (2011) 772-788.

[31] A.L. Becker, A.P.R. Johnston, F. Caruso, Small 6 (2010) 1836-1852.

[32] P.K. Deshmukh, K.P. Ramani, S.S. Singh, A.R. Tekade, V.K. Chatap, G.B. Patil, S.B. Bari, J. Controlled Release 166 (2013) 294-306.

[33] S. Acharya, S.K. Sahoo, Adv. Drug Delivery Rev. 63 (2011) 170-183.

[34] W. Zauner, M. Ogris, E. Wagner, Adv. Drug Delivery Rev. 30 (1998) 97-113.

[35] S. Łukasiewicz, K. Szczepanowicz, Langmuir 30 (2014) 1100-1107.

[36] G. Parekh, P. Pattekari, C. Joshi, T. Shutava, M. DeCoster, T. Levchenko, V. Torchilin, Y. Lvov, Int. J. Pharm. 465 (2014) 218-227.

[37] M.B. Thomas, K. Radhakrishnan, D.P. Gnanadhas, D. Chakravortty, A.M. Raichur, Int. J. Nanomed. 8 (2013) 267-273.

[38] S.F. Yan, J. Zhu, Z.C. Wang, J.B. Yin, Y.Z. Zheng, X.S. Chen, Eur. J. Pharm. Biopharm. 78 (2011) 336-345.

[39] J. Siepmann, N.A. Peppas, Adv. Drug Delivery Rev. 64 (2012) 163-174.

[40] J. Siepmann, F. Siepmann, J. Controlled Release 161 (2012) 351-362.

[41] F. Cuomo, A. Ceglie, M. Piludu, M.G. Miguel, B. Lindman, F. Lopez, Langmuir 30 (2014) 7993-7999.

[42] F. Szoka, D. Papahadjopoulos, Proc. Nat. Acad. Sci. U.S.A. 75 (1978) 4194-4198

[43] P.L. Ritger, N.A. Peppas, J. Controlled Release 5 (1987) 23-36.

[44] S. Shu, C. Sun, X. Zhang, Z. Wu, Z. Wang, C. Li, Acta Biomater. 6 (2010) 210-217.

[45] S.J. Shu, L. Sun, X.G. Zhang, Z.M. Wu, Z. Wang, C.X. Li, J. Nanopart. Res. 13 (2011) 3657-3670.

[46] S. Koutsopoulos, L.D. Unsworth, Y. Nagai, S. Zhang, Nat. Acad. Sci. U.S.A. 106 (2009) 4623-4628.

[47] R. Pjanović, N. Bošković-Vragolović, J. Veljković-Giga, R. Garić-Grulović, S Pejanović, B. Bugarski, J. Chem. Technol. Biotechnol. 85 (2010) 693-698. 